\title{
Aneurisma de la arteria esplénica. Revisión de dos casos intervenidos quirúrgicamente
}

\author{
C. ABAD, D. MONTESDEOCA-CABRERA, T. SÁEZ-GUZMÁN
}

Servicio de Cirugía Cardiovascular. Hospital Universitario de Gran Canaria Dr. Negrín. Las Palmas de Gran Canaria

\author{
SPLENIC ARTERY ANEURYSM. REVIEW OF TWO SURGICALLY \\ OPERATED CASES
}

\section{RESUMEN}

Entre los aneurismas arteriales, los localizados en la arteria esplénica son de infrecuente aparición en la práctica clínica. La etiología puede ser debida y está en relación con: fibrodisplasia, hipertensión portal, embarazo y arteriosclerosis.

La mayor parte son asintomáticos, otras veces la clínica es por la rotura y perforación del aneurisma en peritoneo libre o en un órgano o estructura vecina. Presentamos dos casos de aneurisma silente en la arteria esplénica en dos pacientes, un varón y una hembra de 57 y 59 años respectivamente. En ambos se hizo el diagnóstico por radiología de abdomen, TAC abdominal y arteriografía.

Los dos se intervinieron quirúrgicamente por laparotomía transversa, practicándose resección completa del aneurisma. En los dos casos el diagnóstico anatomopatológico fue arteriosclerosis. Los pacientes están bien y sin evidencia de aneurisma visceral a los 120 y 38 meses respectivamente de la operación.

Se comentan los aspectos clínicos, diagnósticos y el tratamiento de esta entidad.

PALABRAS CLAVE: Aneurisma arteria esplénica. Cirugía.

\begin{abstract}
Among arterial aneurysms, splenic artery aneurysms are very uncommon in the current practice. The etiologic factors are in relation with: angiodisplasia, portal hypertension, pregnancy and atherosclerosis. The great majority are asymptomatic. Symptomatic cases are in relation with rupture in the peritoneum or in organs or structures of the vecinity. We present two cases of splenic artery aneurysm in a 57 year old man and in a 59 year old woman. In both cases the diagnosis was made by plain abdominal x-ray, abdominal scanner and abdominal aortography. Both cases were surgically treated by transverse laparotomy and total aneurysm resection. The pathology dictamen of the two cases was consistent with atherosclerosis. After 120 and 38 months of followup both patients are doing well and without evidence of splenic artery aneurysm. A review of this entity is exposed, with special reference in the etiology, symptomatology, diagnosis and treatment.
\end{abstract}

KEY WORDS: Splenic artery aneurysm. Surgery.

Abad C, Montesdeoca-Cabrera D, Sáez-Guzmán T. Aneurisma de la arteria esplénica. Revisión de dos casos intervenidos quirurgicamente . An Med Interna (Madrid) 2006; 23: 130-132.

\section{INTRODUCCION}

Los aneurismas viscerales son relativamente infrecuentes, dentro de éstos los más habituales son los de la arteria esplénica que representan alrededor del $60 \%$. Otras localizaciones viscerales más infrecuentes son en la arteria hepática $(20 \%)$, arteria mesentérica superior $(5,5 \%)$, tronco celiaco (4\%), arterias gástricas y gastroepiploicas (4\%), arterias intestinales $(3 \%)$, arterias pancreáticas y pancreático duodenales $(2 \%)$, arteria gastroduodenal $(1,5 \%)$ y muy raramente en la arteria mesentérica inferior (1,2\%) (1). Además de su escasa aparición clínica, su tratamiento ha sido realizado por cirujanos cardiovasculares, cirujanos generales, radiólogos, angiólogos, etc; por lo cual la experiencia global de un equipo es escasa y la experiencia individual de un cirujano puede ser limitada.

Presentamos dos pacientes con un aneurisma silente de la arteria esplénica que fueron tratados quirúrgicamente con éxito en un Servicio de Cirugía Cardiovascular. El primer caso ha sido reportado previamente (2). 


\section{CASOS APORTADOS}

Caso 1. Paciente de 57 años y sexo masculino. Entre sus antecedentes personales figuraba el ser fumador de 10 cigarrillos/día, bebedor moderado, fibrilación auricular crónica de 5 años de evolución y padecía de hipertensión arterial en tratamiento con medicación beta-bloqueante. Sufrió una crisis hipertensiva severa que requirió ingreso hospitalario. A su ingreso la tensión arterial era de 220/130mm Hg. En la exploración física el pulso era irregular a 80 lat/min. La exploración cardiológica y abdominal eran normales. La radiografía de tórax, la analítica básica de sangre y orina y temperatura axilar normal. Se instauró tratamiento médico normalizándose las cifras tensionales. En la radiografía simple de abdomen destacaba una calcificación irregular en el hipocondrio izquierdo. Posteriormente se realizó TAC abdominal y arteriografía abdominal (Fig. 1) que mostraba la imagen de un aneurisma en el tercio medio de la arteria esplénica. Se planteó la extirpación quirúrgica. El 3 de Mayo de 1995 se realizó una laparotomía transversa alta por encima del ombligo. Se procedió a la sección entre ligaduras del epiplon gastro-cólico y localización del aneurisma en la parte media de la arteria esplénica que estaba parcialmente alojado en el páncreas y que medía unos $5 \times 5 \times 5 \mathrm{~cm}$. Tras disección se llevó a cabo la ligadura proximal y distal del aneurisma resecándolo al completo. Además se efectúo esplenectomía y resección de cola y parte del cuerpo del páncreas englobados en el proceso. Apendicectomía asociada. Curso postoperatorio sin incidentes, siendo alta a los 9 días de la intervención. La pieza quirúrgica fue enviada a Anatomía Patológica siendo el dictamen de aneurisma esplénico arteriosclerótico; páncreas, bazo y apéndice normales. A los 10 años de la intervención el paciente está bien de la cirugía practicada. Se ha practicado una ecografía abdominal que es normal.

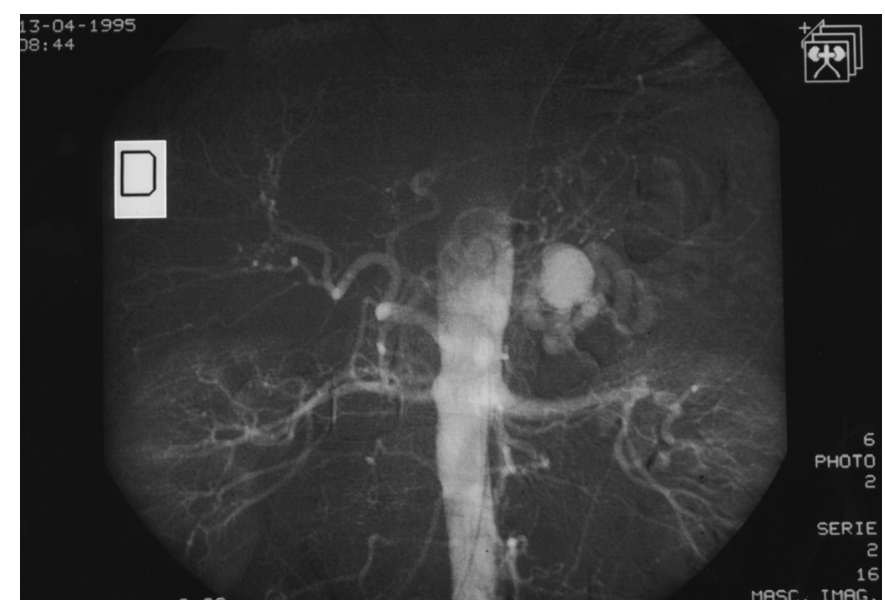

Fig. 1. Aortografía abdominal donde se visualiza aneurisma de la arteria esplénica.

Caso 2. Paciente del sexo femenino y 59 años de edad. En una radiografía simple de abdomen practicada por su médico de cabecera se detectó una masa redondeada y calcificada en flanco izquierdo que fue inicialmente etiquetada de posible aneurisma de la arteria esplénica, por lo que se envió a la paciente a evaluación a cirugía. Se realizó un TAC abdominal (Fig. 2) y angio-RMN de aorta abdominal que confirmó el diagnóstico de aneurisma de la arteria esplénica cerca del hilio del bazo. La enferma estaba asintomática y en sus antecedentes personales destacaba: diabetes mellitus no-insulinodependiente, artrosis de columna lumbar, radiculalgia en extremidades inferiores, operada de estenosis de canal medular en 1995 y dolor intermitente en fosa iliaca derecha de 5 años de evolución. La exploración física era normal, así como la radiografía de tórax, ECG y analítica general básica. Tensión arterial de 130/80 mmHg y temperatura axilar de $36,6^{\circ} \mathrm{C}$.

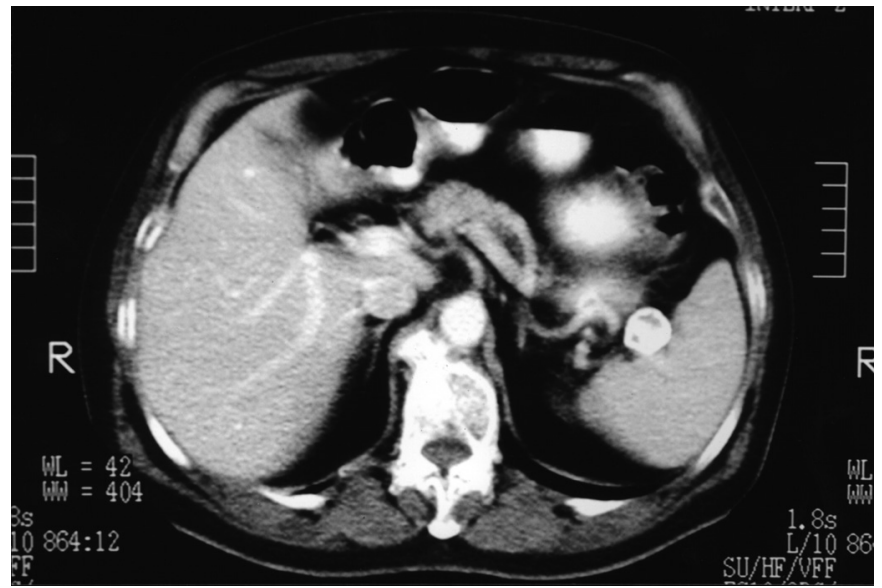

Fig. 2. TAC abdominal con imagen de aneurisma de la arteria esplénica cerca del hilio del bazo.

Con el diagnóstico de aneurisma de arteria esplénica se indicó cirugía. El 19 de marzo de 2002 fue intervenida quirúrgicamente. Se efectúo una laparotomía transversa y sección del ligamento gastroesplénico y espleno-cólico, localizando fácilmente un aneurisma de $2,5 \times 2,5 \mathrm{~cm}$ en la arteria esplénica pegado prácticamente al hilio esplénico. Se realizó liberación quirúrgica del bazo seccionando los ligamentos freno-esplénicos, espleno-renal, vasos cortos y adherencias. A continuación se aisló arteria y vena esplénica y esplenectomía. Además se hizo apendicectomía por los antecedentes de dolor en fosa iliaca derecha. La paciente toleró bien el procedimiento siendo alta en buen estado a los 8 días de la intervención. La pieza extraida se envió a Anatomía Patológica, siendo el diagnóstico de aneurisma de arteria esplénica de 2,5x2,5 cm, de paredes calcificadas y trombo mural de etiología arteriosclerótica. El bazo y el apéndice eran normales. A los 3 años y 2 meses de la intervención la paciente se encuentra en buen estado general. La ecografía abdominal es normal y no hay datos de aneurisma visceral o de otro tipo.

\section{DISCUSIÓN}

La incidencia de los aneurismas de la arteria esplénica es difícil de evaluar pero se puede cifrar en torno al $1 \%$ en necropsias y estudios arteriográficos. Son más frecuentes en adultos y en el sexo femenino. Los factores etiológicos más importantes son: fibrodisplasia de la arteria esplénica, hipertensión portal con esplenomegalia, embarazo y arteriosclerosis (1). Con respecto a la arteriosclerosis puede ser primaria de la arteria esplénica o tratarse de cambios arterioscleróticos y calcificaciones parietales secundarias en el saco aneurismático (1). La mayor parte son clínicamente silentes, como los dos casos aquí presentados, otras veces los pacientes relatan dolor inespecífico en epigastrio e hipocondrio izquierdo.

Al igual que el resto de los aneurismas arteriales el mayor peligro es la rotura. Dicha rotura puede ser a: a) Peritoneo libre con hemoperitoneo y shock hemorrágico o bien rotura con hemorragia localizada inicialmente en la transcavidad de los epiplones y que posteriormente por el hiato de Winslow puede comunicarse con el peritoneo libre, dando lugar a lo que se conoce como rotura en dos tiempos. b) Estómago con hematemesis y melenas. c) Colon con melenas y cuadro de hemorragia digestiva baja. d) Conducto de Wirsung lo que ocasiona el hemosuccus pancreaticus que cursa con hemorragia digestiva alta intermitente, dolor cólico abdominal y ele- 
vación de la amilasemia. e) Vena esplénica con fístula arteriovenosa $(1,3)$. Los factores de riesgo de rotura no están bien determinados, no se conocen con exactitud y pueden ser variables oscilando el riesgo de esta complicación, del 0,5\% al $10 \%$. La rotura es más frecuentes en mujeres embarazadas. Los aneurismas con paredes calcificadas también pueden romperse. La mortalidad en casos de rotura es alta del $25 \%$ al $50 \%$ y en embarazadas hasta del $75 \%$.

El diagnóstico muchas veces se hace por la presencia de una calcificación redondeada en la zona de la arteria esplénica, hecho que acontece en un $70 \%$ de los casos (1) y presente en nuestros dos operados. El diagnóstico más exacto lo proporciona el TAC abdominal, aortografía abdominal y angio-RMN de aorta abdominal y ramas viscerales (1). Dada la posibilidad de rotura y la alta mortalidad de esta complicación con o sin tratamiento, los aneurismas esplénicos deben tratarse. Los aneurismas rotos y sintomáticos, se tratan de forma urgente y preferente respectivamente. Los aneurismas asintomáticos o silentes también deben intervenirse. La mayor parte de estos aneurismas tienen un diáme-

\section{Bibliografía}

1. Zelenock GB, Stanley JC. Splachnic artery aneurysms. In Rutherford RB editor. Vascular Surgery. Fith Edition. Philadelphia. WB Saunders; 2000: p.1369-82.

2. Abad C, Ray V, Ponce G, Macías A, Gómez E, Jiménez P. Aneurisma de arteria esplénica en un paciente con hipertensión arterial. Patología Vascular 1996: 2: 10-14.

3. Abad C, Botey A, López-Pedret J, Mulet J. Aneurysm of the gastroduodenal artery. Surgical treatment in an asymptomatic case. Thorac Cardiovasc Surg 1985; 33: 188-90.

4. Chiesa R, Astore D, Guzzo G, Frigorio S, Tschomba Y, Castellano R, et al. Visceral artery aneurysms. Ann Vasc Surg 2005: 19: 42-8.

5. Reardon PR, Otah E, Craig ES, Mathews BD, Reardon MJ. Laparoscopic tro de $2 \mathrm{~cm}$ (1) y a partir de esta cifra consideramos indicada la intervención.

En el momento actual se contemplan básicamente los siguientes tratamientos: 1) laparotomía y exéresis (4), resección o exclusión del aneurisma por laparoscopia (5) y 3) terapéutica percutánea mediante la implantación de un stent recubierto (6) o bien la embolización (7). Cada modalidad terapéutica tiene sus defensores. La vía clásica, abierta o por laparotomía es un método de probada eficacia $(1-3,8,9)$, pero relativamente traumático y con morbilidad. La vía laparoscópica es una alternativa excelente y poco agresiva, pero requiere de un cirujano experto en cirugía endoscópica. El tratamiento percutáneo endoluminal tiene los inconvenientes de dificultad en la colocación del stent debido a arteria esplénica tortuosa y la probabilidad de recidiva o exclusión aneurismática incompleta. Nosotros consideramos el tratamiento quirúrgico por vía abierta o mejor endoscópica, la mejor opción terapéutica en pacientes con riesgo quirúrgico razonable. Los pacientes con riesgo quirúrgico muy elevado o contraindicación a la cirugía pueden beneficiarse del tratamiento percutáneo endovascular. resection of splenic artery aneurysms. Surg Endosc 2005; 19: 488-93

6. Mayor HR, Hivamoto JS, Wilson MW, Roddy P, Messina LM, Schneider DB. Stent-graft repair of a splenic artery aneurysm. J Vasc Surg 2005; 41: 897.

7. Kasirajan K, Greenberg RK, Chair D, Ouriel K,. Endovascular management of visceral artery aneurysm. J Endovasc Ther 2001; 8: 150-5.

8. Grego FG, Lepidi S, Ragazzi R, Jurilli V, Stnamona R, Deriu GP. Visceral artery aneurysms: a single center experience. Cardiovasc Surg 2003; 11: $19-25$.

9. Sossa C, Tinelli G, Povar P, Aubont A, Thony F, Magre JL. Treatment of visceral artery aneurysms: description of a retrospective series of 42 aneurysms in 34 patients. Ann Vasc Surg 2004; 18: 695-703. 